\title{
Análise de Variáveis Estratégicas para o Desenvolvimento da Agropecuária da Região do Pontal do Paranapanema
}

\author{
Ricardo Firetti ${ }^{1}$, Luiza Maria Capanema ${ }^{2}$, Cristina Fachini ${ }^{3}$, \\ Patrícia Helena Nogueira Turco ${ }^{4}$ e Alceu de Arruda Veiga Filho ${ }^{5}$
}

\begin{abstract}
Resumo: $\mathrm{O}$ objetivo do trabalho é identificar as atividades rurais com maior capacidade de geração de emprego e renda e as relações existentes entre o desenvolvimento científico e capacitação rural no Pontal do Paranapanema em horizonte temporal de 10 anos (2008-2018). Utilizou-se a abordagem Technological Foresight para a estruturação do levantamento prospectivo e métodos e técnicas de estatística multivariada na análise dos resultados. As informações foram levantadas em um painel estruturado com a participação de 50 especialistas da região. Os resultados demonstram que níveis de capacitação técnica, oferta de tecnologias e assistência técnica são variáveis que têm grande importância para geração de emprego e renda futura. Além disso, ações de transferência de tecnologia são prioritárias no curto prazo e as de P\&D com capacitação técnica de produtores e trabalhadores rurais, no longo prazo. Destacaram-se, para a geração de empregos ao trabalhador rural, as atividades de produção relacionadas à agroindústria. Novas atividades como a heveicultura, eucalipto, caprinos, ovinos, integração lavoura-pecuária e plantas ornamentais foram consideradas importantes para a geração de renda.
\end{abstract}

Palavras-chaves: agricultura familiar, produtor rural, prospecção, technological foresight, transferência de tecnologia.

Abstract: The objective of this study is to identify rural activities with greater capacity to generate employment and income, and the relationship between scientific development and rural training in the Pontal do Paranapanema region in a period of 10 years (2008-2018). We have used the Technological Foresight approach to structure the survey and prospective methods and techniques of multivariate statistical analysis of results. Information has been raised at a panel organized with the participation of 50 experts from the region, and the

1 Pesquisador da Apta Regional (DDD/APTA/SAA-SP). E-mail: rfiretti@apta.sp.gov.br

2 Pesquisadora do Instituto Agronômico (IAC/APTA/SAA-SP). E-mail: luiza@apta.sp.gov.br

3 Pesquisadora da Apta Regional (DDD/APTA/SAA-SP). E-mail: cfachini@apta.sp.gov.br

4 Pesquisadora da Apta Regional (DDD/APTA/SAA-SP). E-mail: patricia.turco@apta.sp.gov.br

5 Pesquisador da Apta Regional (DDD/APTA/SAA-SP). E-mail: alceu@apta.sp.gov.br 
results showed that levels of technical expertise, supply of technologies and technical assistance are variables which are important to generate employment and future income. In addition, actions of technology transfer are essential in the short run and the RED with technical training for farmers and workers, in the long run. Production activities related to agribusiness were highlighted for job creation to rural workers. New activities, such as rubber tree, eucalyptus, goats, sheep, crop-livestock integration and ornamental plants were considered important to generate income.

Key-words: family farms, rural farmer, prospecting demands, technological foresight, technology transfer.

Classificação JEL: J43, O32, Q16.

\section{Introdução}

O estado de São Paulo é reconhecido nacionalmente como a unidade da Federação com melhores índices de desenvolvimento urbano e rural. Apresenta a maior densidade demográfica do País e abriga a mais complexa região metropolitana. Tem importância fundamental para a economia do Brasil ao responder por parte expressiva (34\%) do Produto Interno Bruto. Sua participação é representativa na produção agropecuária, industrial e no setor terciário. Além disso, é o principal centro exportador nacional.

Entretanto, a $10^{\mathrm{a}}$ Região Administrativa (RA) do estado de São Paulo, cuja sede é o município de Presidente Prudente, é considerada uma das últimas fronteiras do desenvolvimento paulista, com presença constante nos noticiários que tratam de conflitos pela posse de terras. Localizada no extremo oeste do estado, a região abrange 53 municípios que ocupam uma área de $23.953 \mathrm{~km}^{2}$ e abriga 820,9 mil habitantes; é caracterizada por ser uma das regiões menos densamente povoadas do estado (34,3 habitantes por $\left.\mathrm{km}^{2}\right)$, estando à frente somente da Região Administrativa (RA) de Registro $\left(23,6 \mathrm{hab} / \mathrm{km}^{2}\right)$. A maioria dos municípios da região é de pequeno porte e mais da metade possui população inferior a 10.000 habitantes.

A Secretaria de Economia e Planejamento do Estado de São Paulo (SÃO PAULO, 2008) apontou que um importante desafio para esta região é a diversificação agropecuária com desenvolvimento de novas atividades econômicas geradoras de emprego e renda que possam auxiliar na fixação da população na RA, considerando-seque alguns de seus municípios apresentaram taxas de crescimento populacional negativa entre 2000 e 2005.

Para Veiga (2001), a diversificação produtiva (seja ela primária, secundária ou terciária) levaria a uma base econômica mais flexível e menos sujeita às oscilações de um único mercado, enquanto a especialização induziria à formação de uma economia local que reagiria apenas aos sinais enviados por um determinado mercado. $\mathrm{O}$ autor ainda acrescenta que, para a geração de empresas, empregos e renda em regiões com predomínio da agricultura familiar, como é o caso da Região Administrativa de Presidente Prudente, seria preciso a disponibilização de um mínimo de condições favoráveis em termos de comunicações e de serviços e, sobretudo, de condições para o desenvolvimento de uma cultura de empreendedorismo e inovação ${ }^{6}$.

O Pontal do Paranapanema integra a Região Administrativa de Presidente Prudente, em sua porção no extremo-oeste, fazendo divisa concomitantemente aos estados de Mato Grosso do Sul e Paraná, sendo banhado pelos rios Paraná e Paranapanema. Formada por 32 municípios, a região concentra quase a totalidade dos assentamentos rurais da Região Administrativa e sua estrutura fundiária é caracterizada por pequenas propriedades.

Os municípios que compõem o Pontal do Paranapanema abrangem 1,85 milhão de

6 Para uma discussão sobre empreendedorismo, incubadoras e agricultura familiar, ver Fachini et al. (2006). 
hectares $(7,4 \%$ do total estadual), dos quais 91,2\% eram ocupados por Unidades de Produção Agropecuária (UPA), conforme o Levantamento Censitário de Unidades de Produção Agrícola do Estado de São Paulo, realizado no biênio 2007/2008 (SÃO PAULO, 2009).

Em 2007/2008, 20.950 UPAs existiam no Pontal do Paranapanema, das quais $79,4 \%$ correspondiam a propriedades com área de 50 hectares (ha) e ocupavam $17,21 \%$ da área total de UPAs. No estrato de 50 a500 hectares, sumarizou-se significativa parcela de $33,4 \%$ da área total, correspondendo a $17,7 \%$ das unidades. As propriedades que têm acima de 500 hectares (0,6\% das UPAs) ocupavam 49,41\% da área total (SÃO PAULO, 2009).

A ideia da realização de prospecção de demandas tecnológicas para a transferência de tecnologia para o setor produtivo é antiga (JANTSCH, 1967), porém, ganhou nova abordagem, uma vez que a relação entre pesquisa e desenvolvimento e inovação não é linear e envolve um número muito grande de atores que atuam de diferentes maneiras no processo de produção dos produtos rurais.

A utilização de metodologias de prospecção de demandas ganhou mais relevância em instituições de pesquisa agropecuária no Brasil a partir da década de 90, com ampla difusão pela Embrapa (Empresa Brasileira de Pesquisa Agropecuária). A partir desse período, a Agência Paulista de Tecnologia dos Agronegócios (Apta) desenvolveu estudos em algumas regiões do estado: Sudoeste Paulista (NOGUEIRA e MELLO, 2001; NOGUEIRA et al., 2005), Médio Paranapanema (ROCHA et al. 2005) e Vale do Paraíba (CAPANEMA et al., 2006). Além dos estudos regionais, existem trabalhos realizados focados em cadeias produtivas específicas (BLISKA et al., 1998, 2005, 2007). Os resultados dos estudos prospectivos com foco em regiões revelam e priorizam as cadeias produtivas de maior interesse e maior potencial para a região estudada e aqueles direcionados às cadeias produtivas específicas permitem estruturação de ações de governança nas cadeias. Ambos os enfoques fornecem subsídios para organizações de ciência e tecnologia traçarem diretrizes e realizarem ações de gestão de ciência, tecnologia e inovação (C,T\&I).

O trabalho está organizado em três seções além desta introdução. Na primeira é apresentada a metodologia da pesquisa, destacando-se a abordagem usada para estruturação do levantamento das informações e as técnicas de estatística multivariada utilizada para análise dos resultados. Na seção seguinte, é disponibilizada a análise dos resultados. Esta seção contempla dois itens, sendo que, no primeiro, é realizada uma análise, com base em técnicas de estatística multivariada de variáveis selecionadas compreendendo: teste de validação da amostra (alpha de crombach), análise fatorial pelo método dos componentes principais e uma análise de correspondência (análise interpretativa). No segundo, é apresentada uma análise de agrupamento (K-means cluster) que permitiu a seleção de atividades a partir de uma lista existente, considerando-se as variáveis: geração futura de empregos ao trabalhador rural e geração futura de renda ao pequeno produtor rural (análise indicativa). Na última seção são feitas as considerações finais.

\section{Metodologia de pesquisa}

O estudo foi realizado na região do Pontal do Paranapanema, composto por 32 municípios ${ }^{7}$, e que se constitui na área de abrangência do Polo Regional de Desenvolvimento Tecnológico dos Agronegócios da Alta Sorocabana, divisão de pesquisa e desenvolvimento da Apta.

\footnotetext{
7 Constituem o Pontal do Paranapanema os municípios de Alfredo Marcondes, Álvares Machado, Anhumas, Caiabu, Caiuá, Emilianópolis, Estrela do Norte, Euclides da Cunha Paulista, Iepê, Indiana, João Ramalho, Marabá Paulista, Martinópolis, Mirante do Paranapanema, Nantes, Narandiba, Piquerobi, Pirapozinho, Presidente Bernardes, Presidente Epitácio, Presidente Prudente, Presidente Venceslau, Rancharia, Regente Feijó, Ribeirão dos Índios, Rosana, Sandovalina, Santo Anastácio, Santo Expedito, Taciba, Tarabai, e Teodoro Sampaio.
} 
Tabela 1. Distribuição dos participantes no painel de especialistas em função de sua origem institucional.

\begin{tabular}{lcc}
\hline \multicolumn{1}{c}{ Instituições } & Frequência & Freq. Relativa \\
\hline Instituições Públicas de Pesquisa & 17 & 0,34 \\
Universidades Públicas e Privadas & 4 & 0,08 \\
Segmento Rural & 12 & 0,24 \\
Transferência de Tecnologias & 9 & 0,18 \\
Executivo Público & 5 & 0,10 \\
Segmento Empresarial & 3 & 0,06 \\
Total & $\mathbf{5 0}$ & $\mathbf{1 , 0 0}$ \\
\hline
\end{tabular}

Fonte: Dados da pesquisa.

Para identificar as atividades com potencial futuro de geração de empregos e renda, considerando-se aqui o conceito de agronegócios desenvolvido por Davis e Goldberg (1957), foi realizado um levantamento de dados a partir de um painel estruturado com 50 especialistas do Pontal do Paranapanema (Tabela 1), utilizando-se a abordagem de prospeç̧ão de futuro denominada Technological Foresight (HORTON, 1999; GODET, 2000),que ébaseada em processos participativos e que permite a formação de consenso a partir de vários pontos de vista sobre as possíveis formas desejáveis nas quais o futuro poderia se desenvolver.

No painel, realizado em dezembro de 2007, os respondentes se dividiram em quatro grupos, sendo que cada grupo foi instruído a priorizar atividades rurais mais importantes para o desenvolvimento regional.

Além das respostas consensuais por grupo, procedeu-se uma avaliação, na qual os participantes apresentaram respostas individuais a partir da utilização de uma escala Likert de cinco posições, compreendendo notas de 1 a 5 (muito baixo a muito alto). Com a utilização da escala buscou-se quantificar a percepção dos atores sobre a capacidade atual na geração de empregos e renda para o trabalhador (variável 1); a capacidade futura na geração de empregos e renda para o trabalhador (variável 2); a capacidade atual na geração de renda para o pequeno produtor (variável 3), a capacidade futura na geração de renda para o pequeno produtor (variável 4); a capacidade atual na geração de renda para o médio e grande produtor (variável 5); a capacidade futura na geração de renda para o médio e grande produtor (variável 6); o atual nível de capacitação regional (variável 7); o futuro nível de capacitação regional (variável 8); a contribuição atual ao desenvolvimento científico e tecnológico regional (variável 9); a contribuição futura ao desenvolvimento científico e tecnológico regional (variável 10); a disponibilidade atual de assistência técnica (variável 11), a eficiência e efetividade atual dos mecanismos de Transferência de Tecnologia do polo à região do Pontal do Paranapanema (variável 12) e, por fim, sobre a oferta atual de tecnologia (variável 13).

As variáveis selecionadas foram submetidas à análise estatística multivariada utilizando o software STATISTICA 5.0 (STATSOFT INC., 1997) compreendendo: teste de validação da amostra (alpha de crombach), análise fatorial pelo método dos componentes principais, análise de correspondência e análise de agrupamento ( $k$-means cluster e two way joinning).

No que se refere ao tamanho da amostra, Hair et al. (2005) apontam que dificilmente se realiza uma análise fatorial com menos de 50 observações mantendo uma proporção em torno de 10 variáveis para cada observação (ator representativo). Outra questão a ser destacada quanto aos critérios de significância é a necessidade de utilização de cargas fatoriais acima de $\pm 0,70$, pois são consideradas indicativas de estrutura bem definida sendo a meta de qualquer análise fatorial.

Outro item questionável é retratado pela eventual heterogeneidade dos respondentes 
e sua eventual influência na análise, contudo, novamente segundo Hair et al. (2005), caso seja realmente heterogênea a pelo menos um subconjunto das variáveis, então, os primeiros fatores representam aquelas variáveis mais homogêneas em toda amostra. Deve-se observar, ainda, que no caso deste estudo, embora os atores envolvidos tenham funções econômicas heterogêneas na região (heterogêneos), todos atuam há muitos anos diretamente com o setor agropecuário do Pontal do Paranapanema (homogeneidade).

\subsection{Teste de validação da amostra}

O teste de Alpha de Crombach fornece um índice que varia de 0 a 1 . Quanto mais próximo de 1 , mais eficazes são as variáveis que estão sendo testadas. Hair et al. (2005), aconselham que um coeficiente abaixo de 0,7 caracteriza um conjunto de variáveis fracas. Em síntese, o teste permite medir a correlação existente entre as variáveis, podendo sofrer distorção em decorrência do número de variáveis envolvidas. Não é aconselhado o emprego de menos de cinco (5) variáveis para obtenção do teste.

A proporção da variância verdadeira pode ser estimada pela comparação entre a soma das variâncias com a variância da soma. Assim, pode-se calcular:

$$
\alpha=\left(\frac{k}{k-1}\right) \times\left[1-\frac{\sum S_{i}^{2}}{S_{\text {soma }}^{2}}\right]
$$

Em que:

k indica o número de itens (ou questões);

$\mathrm{s}_{\mathrm{i}}{ }^{2}$ indica a variância para cada um dos itens (ou a variância das notas de cada uma das questões) e; $\mathbf{s}^{2}{ }_{\text {soma }}$ é a variância da soma de todos os itens (ou a variância das notas do exame).

Esta fórmula define o índice mais comum de confiabilidade, o coeficiente alpha de Cronbach. Se não houver nenhum componente real nas notas, então, a variância da soma será igual à soma das variâncias individuais; dessa forma, o coeficiente $\alpha$ será nulo. Por outro lado, se não houver erros, o coeficiente será igual à unidade.
Quando $\sum s_{1}^{2}>s_{\text {soma }}^{2} \quad$ o coeficiente $\alpha$ é negativo.

Nichols (2007) analisa este aspecto e conclui que isto frequentemente é indicativo da existência de muitos erros no experimento, talvez erros de digitação ou que, porventura, os itens não estejam medindo informações diferentes.

\subsection{Análise fatorial pelo método das componentes principais}

A Técnica de Análise Fatorial por Componentes Principais tem o objetivo de resumir as informações provenientes de diversas variáveis em um número mais reduzido de fatores, que explicam a maior parte da variância total da análise (ALEIXO, 2007).

Hoffman (1992) considera que "na análise de um problema é comum passar a utilizar apenas os primeiros componentes principais, aos quais corresponde, geralmente, grande parte da variância das variáveis. É claro que alguma informação é perdida quando substituímos as diversas variáveis por um número menor de componentes principais. Por outro lado, há vantagens óbvias em substituir um número relativamente grande de variáveis, com problemas de multicolinearidade, por um número relativamente pequeno de variáveis (componentes principais) não correlacionadas".

O método de análise fatorial pode ser expresso na forma matemática (HAIR et al., 2005) através de uma combinação linear entre as variáveis $\left(\mathrm{X}_{\mathrm{i}}\right)$ e K fatores comuns (F).

$$
X_{i}=A_{i l} F_{1}+A_{i 2} F_{2}+\ldots+A_{i k} F_{k}+U_{i}+E_{i}
$$

Em que:

$\mathrm{A}_{\mathrm{ik}}$ - Cargas fatoriais. Utilizadas para combinar linearmente os fatores comuns;

$\mathrm{F}_{\mathrm{k}}$ - Fatores comuns;

$\mathrm{U}_{\mathrm{i}}$ - Fator único;

$E_{i}-$ Fator de erro.

As cargas fatoriais indicam a intensidade das relações entre as variáveis normalizadas $X_{i}$ e os fatores. Quanto maior uma carga i fatorial, mais associada com o fator se encontra a variável. 
A variância comum h, ou comunalidade, querepresenta quanto da variância total i de $X$ é reproduzida pelos fatores comuns, é calculada a partir da somatória ao quadrado das cargas fatoriais a partir de $i$. A variância única $U_{i}$ parte da variância total que não se associa com a variância das outras variáveis. O termo E representa o erro de observação, de mensuração i ou de especificação do modelo.

Eventualmente, a interpretação dos fatores pode ser facilitada com o uso da rotação ortogonal de um grupo de variáveis altamente correlacionadas, visando maximizar os valores de coeficientes da correlação entre as variáveis e os fatores calculados. O método VARIMAX, utilizado neste estudo, procura minimizar o número de variáveis fortemente relacionadas com cada fator e aproxima as cargas fatoriais de 1 e 0 , melhorando o potencial de análise (FUENTES LLANILLO, 2006). Assim, permite obter fatores mais facilmente interpretáveis.

\subsection{Análise de correspondência}

A "Análise de Correspondência" (AC) foi proposta em 1960 por Benzécri (1973 apud BOURRUCHE e SAPORTA, 1980), a qual, devido às suas propriedades matemáticas e pela riqueza de suas interpretações, tornou-se um ótimo instrumento para análise qualitativa. É uma técnica multivariada utilizada para examinar variáveis categóricas, por meio de seu cruzamento ou contingenciamento, tomando-se como base a distribuição de massa do conjunto de observações, permitindo a visualização das relações entre linhas e colunas num mesmo espaço gráfico (SILVA e VERDINELLI, 1997). Por meio dela procura-se encontrar as relações recíprocas, as associações e as oposições, entre variáveis ou entre objetos e variáveis (BENZÉCRI, 1973).

Neste trabalho, para auxiliar na interpretação da análise, os dados serão dispostos em gráfico com escores para localização no sistema plano normalizado canônico, um método que utiliza a normalização por linhas e colunas simultaneamente. A Análise de Correspondência será utilizada para auxiliar na explicação dos fatores extraídos, maximizando a interpretação da análise de fatores por componentes principais.

\subsection{Análise de Agrupamento: \\ k-means cluster e two-way joinning}

O método de agrupamento k-means cluster é dito nãohierárquico por repartição; consiste em um procedimento no qual, dado um número de clusters previamente determinado, calcula pontos que representam os "centros" destes clusters e que são espalhados homogeneamente no conjunto de respostas obtidas através do questionário, e movidos heuristicamente até alcançar um equilíbrio estático (HAIR et al., 2005).

Procede-se, em seguida, uma divisão de todos os casos obtidos pelos ' $\mathrm{k}$ ' grupos preestabelecidos,e a melhor partição dos ' $\mathrm{n}$ ' casos será aquela que otimize o critério escolhido. Em outras palavras, o procedimento inicia-se usando os valores dos primeiros $\mathrm{k}$ casos como estimativas temporárias das médias dos clusters.

O critério de agrupamento do K-means pode ser descrito como sendo:

$$
E=\sum_{k=1}^{K} \sum_{x_{i} \in C_{k}} d\left(x_{i}, x_{0 k}\right)
$$

Em que:

$\mathrm{X}_{0 \mathrm{k}}$ é o centroide do cluster $\mathrm{C}_{\mathrm{k}}$; $\mathrm{d}\left(\mathrm{X}_{\mathrm{i}}, \mathrm{X}_{0 \mathrm{k}}\right)$ é a distancia entre os pontos $X_{\mathrm{i}}$ e $\mathrm{X}_{0 \mathrm{k}}$.

O centroide pode ser a média ou a mediana de um grupo de pontos. O objetivo do K-means é minimizar a distância entre cada ponto e o seu respectivo centroide (HAIR et al., 2005).

Ao aplicar um critério de otimização que divida uma amostra em ' $\mathrm{k}$ ' grupos homogêneos, pretende-se que, dentro de cada grupo, os elementos sejam os mais semelhantes possíveis entre si, ao passo que as semelhanças entre os elementos de grupos distintos sejam as menores possíveis (LOURENÇO e MATIAS, 2000).

Esta técnica foi utilizada para agrupar as atividades indicadas no painel de especialistas, levando-se em consideração as variáveis referentes à geração futura de empregos ao traba- 
lhador rural e geração futura de renda ao pequeno produtor rural. A escolha destas variáveis se deve à consideração da especificidade da estrutura fundiária da região. No Pontal do Paranapanema, em 2007/2008, 79,4\% das Unidades de Produção Agropecuária (UPAs) correspondiam a propriedades com menos de 50 hectares.

O método two-way joining, segundo Rocha et al. (2000), é representado por um gráfico de escala de cores que expressa, na leitura vertical, os diferenciais entre cada variável avaliada pelos especialistas, quão desigual é a avaliação para as diferentes atividades e, na horizontal, uma diferenciação das escalas apontadas dentro de cada atividade. Uma maior variação de cores indica maior heterogeneidade.

Com esta técnica, é possível analisar o agrupamento de casos (atividades) e variáveis simultaneamente por meio de suas similaridades e avaliar, principalmente, a situação atual do aparato para geração e tranferência de tecnologias frente às necessidades de capacitação e contribuição da pesquisa e desenvolvimento apontados pelos especialistas, para que as atividades identificadas possam de fato expressar seu potencial.

\section{Análise de resultados}

\subsection{Análise interpretativa}

O teste de adequação amostral Alfa de Cronbach apresentou valores para as variáveis brutas de 0,95 (índice que varia de 0 a 1), um número bastante elevado.

A análise fatorial realizada com as 13 variáveis, já descritas anteriormente e obtidas a partir do painel de atores representativos, foi efetuada utilizando-se a rotação ortogonal VARIMAX. Contudo, a variável 10 (contribuição futura do desenvolvimento científico e tecnológico regional) foi extraída da análise por possuir cargas fatoriais elevadas em diferentes fatores, assim como preconizado por Hair et al. (2005).

Desta forma, as variáveis foram agrupadas em dois fatores de 12 possíveis (Tabela 2) utilizando, para isso, os critérios da raiz latente (HAIR et al., 2005), que levam em consideração a presença de pelo menos uma variável explicada em cada fator (autovalor maior ou igual a 1).

A partir do segundo fator, as taxas de acréscimo da representação da variância dos dados são cada vez menores, assumindo valores que

Tabela 2. Análise fatorial por componentes principais.

\begin{tabular}{ccccc}
\hline Fatores & Autovalores & \% da variância total & Autovalor acumulado & \% da variância total acumulada \\
\hline 1 & 7,826119 & 65,21766 & 7,826119 & 65,21766 \\
2 & 1,830128 & 15,25107 & 9,656247 & 80,46873 \\
3 & 0,738887 & 6,157389 & 10,39513 & 86,62611 \\
4 & 0,479148 & 3,992898 & 10,87428 & 90,61901 \\
5 & 0,305148 & 2,542897 & 11,17943 & 93,16191 \\
6 & 0,244108 & 2,034232 & 11,42354 & 95,19614 \\
7 & 0,184083 & 1,534024 & 11,60762 & 96,73017 \\
8 & 0,158095 & 1,317461 & 11,76572 & 98,04763 \\
9 & 0,125615 & 1,046792 & 11,89133 & 99,09442 \\
10 & 0,054677 & 0,455644 & 11,94601 & 99,55006 \\
11 & 0,032746 & 0,272887 & 11,97875 & 99,82295 \\
12 & 0,021246 & 0,177049 & 12 & 100 \\
\hline
\end{tabular}

Fonte: Resultados da pesquisa. 
Tabela 3. Coeficientes de correlação entre as variáveis analisadas e os respectivos fatores.

\begin{tabular}{lccc}
\hline & Variáveis & Fator $\mathbf{1}$ & Fator $\mathbf{2}$ \\
\hline Var 1 & Geração empregoao trabalhador & 0,833 & 0,384 \\
Var 2 & Geração emprego ao trabalhador - futuro & 0,237 & 0,918 \\
Var 3 & Geração renda ao pequeno produtor & 0,704 & 0,516 \\
Var 4 & Geração renda ao pequeno produtor - futuro & 0,083 & 0,897 \\
Var 5 & Geração renda médio/grande produtor & 0,814 & 0,471 \\
Var 6 & Geração renda médio/grande produtor - futuro & 0,367 & 0,714 \\
Var 7 & Nível capacitação atual & 0,891 & 0,314 \\
Var 8 & Nível capacitação futuro & 0,365 & 0,819 \\
Var 9 & Contribuição atual da P\&D & 0,925 & 0,111 \\
Var 11 & Disponibilidade de assistência técnica & 0,812 & 0,184 \\
Var 12 & Eficiência do polo em transferência de tecnologias & 0,873 & 0,061 \\
Var 13 & Oferta atual de tecnologias & 0,816 & 0,352 \\
\hline
\end{tabular}

Fonte: Resultados da pesquisa.

contribuem menos à análise. Neste caso, dois fatores representam $80,46 \%$ da variância amostral dos dados. Foram destacados visualmente os coeficientes de correlação superiores a 0,70 entre as variáveis selecionadas e os dois fatores extraídos na análise (Tabela 3).

O Fator 1 explica 65,21\% da variância total e tem correlações significativas com as variáveis: contribuição atual da P\&D (0,925), nível de capacitação atual $(0,891)$, eficiência do polo em transferência de tecnologias $(0,873)$, geração de empregos ao trabalhador rural $(0,833)$, oferta atual de tecnologias $(0,816)$, geração de renda ao médio/grande produtor $(0,814)$, disponibilidade de assistência técnica $(0,812)$ e geração de renda ao pequeno produtor $(0,704)$. Pode-se considerar que este fator representa as condições atuais do Pontal do Paranapanema, com reflexos visíveis na capacidade de geração de empregos e renda.

A análise de múltipla correspondência, entre as variáveis"contribuição atual da P\&D" e aquelas referentes ao arcabouço de transferência de tecnologias ao Pontal do Paranapanema (Figura 1), aponta para a convergência das classes

Figura 1. Análise de correspondência entre as variávies ligadas à geração e transferência de tecnologias.

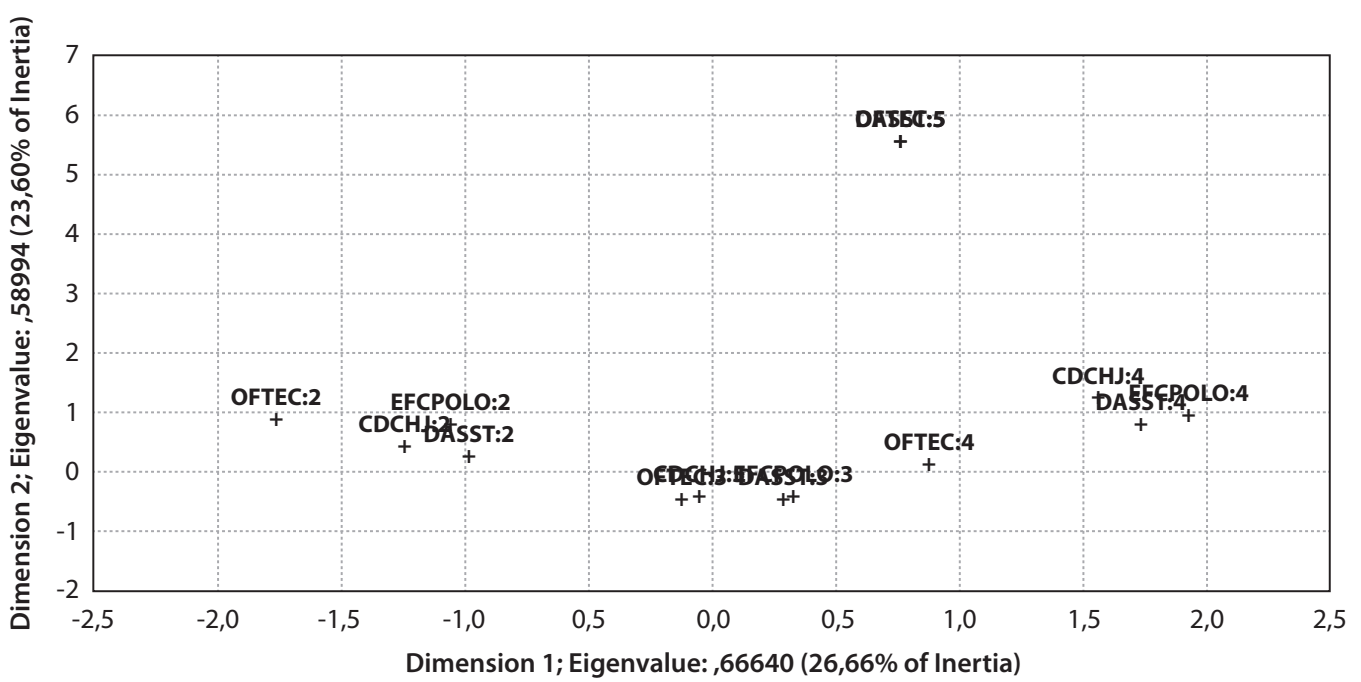

Legenda: CDCHJ - contribuição atual do desenvolvimento científico; OFTEC - oferta de tecnologias; DASS - disponibilidade de assistência técnica; EFCPOLO - eficiência do polo em transferir tecnologias.

Fonte: Resultados da pesquisa. 
Figura 2. Análise de correspondência entre as variáveis ligadas ao nível de capacitação rural e capacidade de geração de renda a produtores rurais.

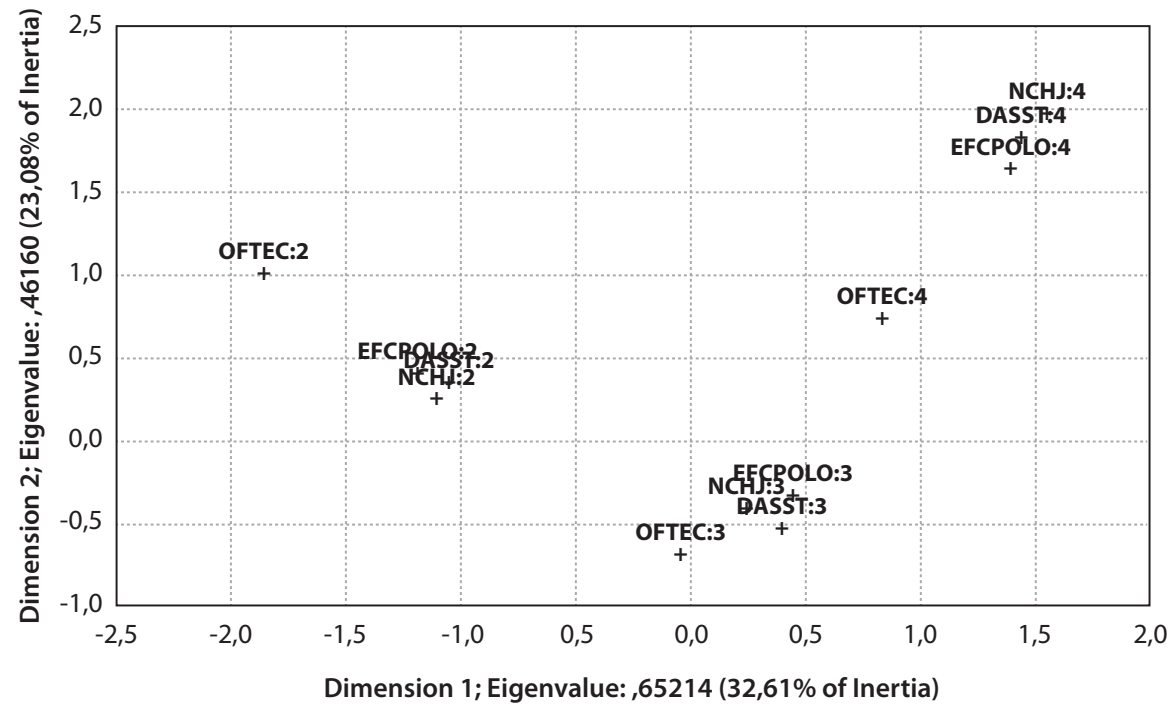

Legenda: NCHJ - nível de capacitação atual; OFTEC - oferta de tecnologias; DASS - disponibilidade de assistência técnica; EFCPOLO - eficiência do polo em transferir tecnologias.

Fonte: Resultados da pesquisa.

de resposta e agrupamento das pontuações. A partir deste gráfico entende-se que, na avaliação dos especialistas, o nível de contribuição ao desenvolvimento científico e tecnológico das instituições regionais tem correspondência direta com a avaliação realizada sobre a oferta de tecnologias, a disponibilidade de assistência técnica e a eficiência do polo em transferir tecnologias, principalmente para as classes de pontuação 2 e 3 (baixo e médio).

Por sua vez, a concentração dos esforços de P\&D e, principalmente, de transferência de tecnologias em atividades específicas tem seu reflexo no nível de capacitação rural da região. Observa-se na análise de correspondência múltipla,apresentada na Figura 2, que os níveis mais elevados de pontuação na variável de capacitação rural atual (NCHJ4) correspondem aos níveis mais elevados de disponibilidade de assitência técnica (DASST4) e eficiência do polo regional em transferência de tecnologias (EFCPOLO4). Para as escalas 2 e 3 das variáveis analisadas, ocorre ainda correspondência maior com a variável oferta de tecnologias (OFTEC).
A análise de múltipla-correspondência aponta a influência positiva que as atividades de geração e transferência de tecnologias realizadas pelo polo regional podem ter na capacidade de geração de empregos e renda (Figura 3).

A partir do gráfico é possível observar que as atividades classificadas como "alta" e "muito alta" capacidade de geração de empregos aos trabalhadores rurais (GETRAB4 e GETRAB5) e de renda aos pequenos produtores (GRPP4) têm correspondência, ou seja, podem ter sido influenciadas pela alta contribuição ao desenvolvimento científico e tecnológico $(\mathrm{CDCHJ} 4)$ e eficiência do polo regional em transferir tecnologias (EFCPOLO4).

Este fato é reforçado pela correspondência existente entre as demais classes de resposta das variáveis, em que a baixa eficiência e contribuição estão próximas de valores semelhantes de capacidade de geração de empregos e renda.

No Fator 2, que concentra 15,25\% da variância total, encontram-se as variáveis relacionadas à "geração de empregos ao trabalhador rural no 
Figura 3. Análise de correspondência entre variáveis de contribuição científica e transferência de tecnologias do polo regional e variáveis de capacidade de geração de empregos e renda.

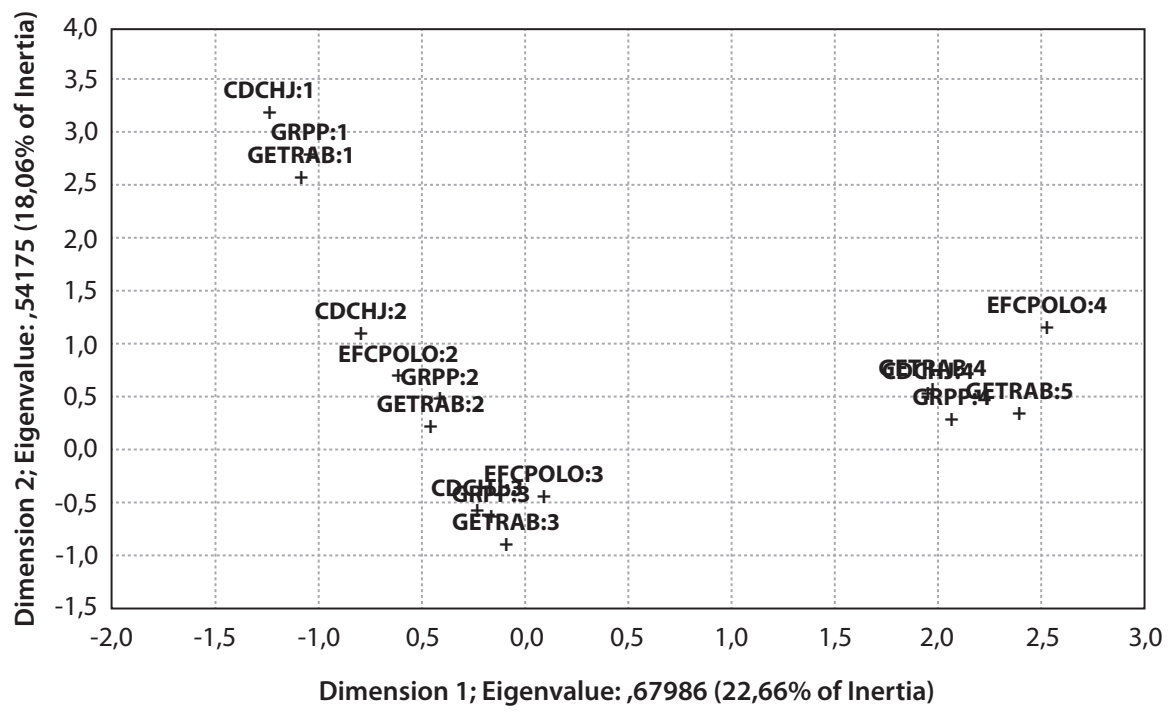

Legenda: $\mathrm{CDCHJ}$ - contribuição atual do desenvolvimento científico; EFCPOLO - eficiência do polo em transferir tecnologias; GETRAB - geração de emprego ao trabalhador rural; GRPP - geração de renda ao pequeno produtor.

Fonte: Resultados da pesquisa.

Figura 4. Análise de correspondência entre as variáveis ligadas ao nível de capacitação rural e capacidade de geração de renda a produtores rurais.

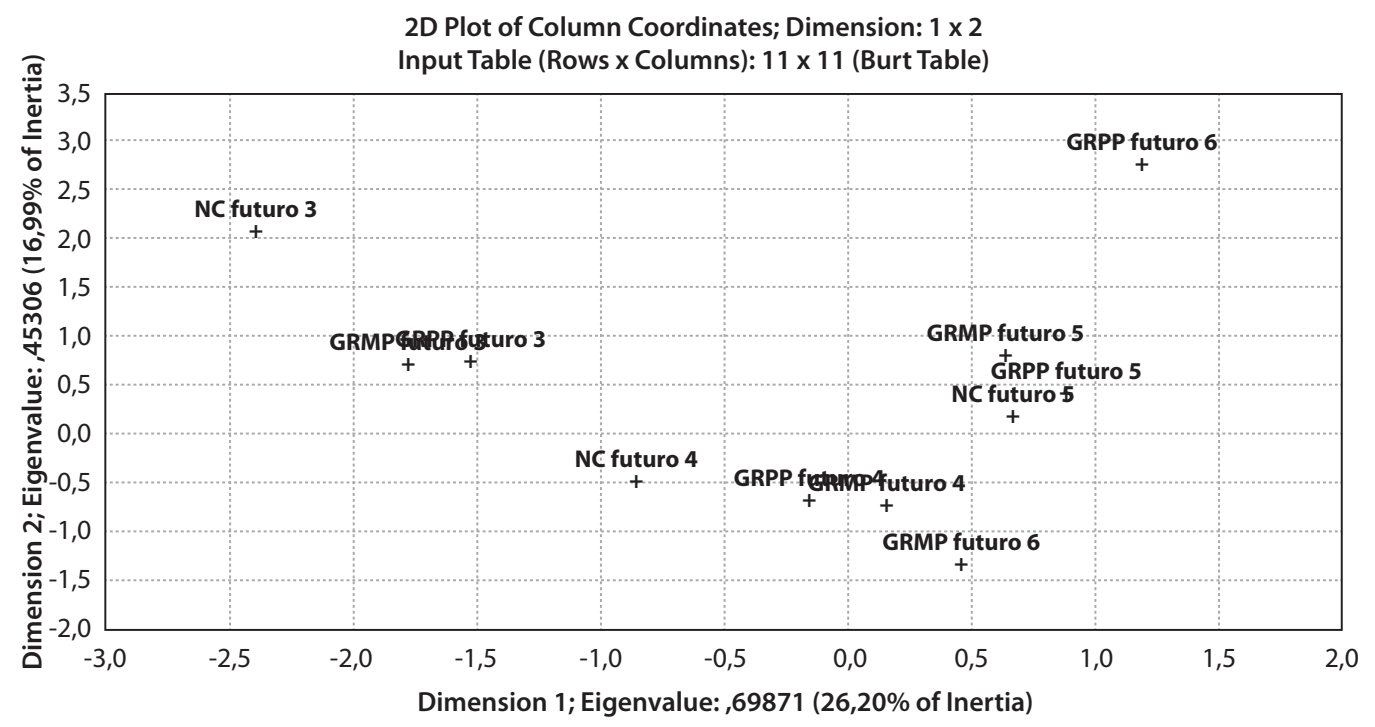

Legenda: GRMP futuro - geração de renda ao médio e grande produtor (no futuro); GRPP futuro - geração de renda ao pequeno produtor (no futuro); NC futuro - necessidade de capacitação (no futuro).

Fonte: Resultados da pesquisa. 
futuro" (0,918), geração de renda ao pequeno produtor rural no futuro $(0,897)$, nível de capacitação no futuro $(0,819)$ e geração de renda ao médio e grande produtor no futuro.

O fator expressa o potencial regional para a geração de empregos e renda. Nessa perspectiva, nota-se a importância dada para a necessidade de capacitação rural, o que torna estratégico este tema regionalmente no horizonte de 10 anos, uma vez que tal questão é resolvida de forma ineficiente nos dias atuais.

A análise de correspondência múltipla entre as variáveis do Fator 2 (Figura 4) aponta para uma forte correlação entre os diferentes níveis de capacitação futura e a geração futura de renda para os produtores rurais, indicando que o potencial gerador econômico das atividades apresentadas no painel de especialistas somente poderá materializar-se caso haja íntima relação com a capacitação dos envolvidos.

Isto exigirá esforços de agentes públicos e privados da região, de alguma forma ligados a esse processo, para quehaja uma adequação dos processos de capacitação dos diversos atores com o objetivo de atender satisfatoriamente essas necessidades.
O painel de especialistas apontou também a necessidade de existência de uma relação próxima, no futuro, entre a contribuição do desenvolvimento científico e tecnológico e o nível de capacitação exigido; este último, por sua vez, relacionado com a geração de renda para produtores rurais.

A Figura 5 apresenta a análise de correspondência entre essas variáveis, em que se observa a existência de correlação entre os níveis de capacitação exigidos no futuro e a contribuição do sistema de pesquisa e desenvolvimento. $\mathrm{Ou}$ seja, quanto mais elevado o nível de capacitação, maior a importância da contribuição do desenvolvimento científico e tecnológico.

A partir da análise Two-way Joinning é possível identificar, de forma geral, que os painelistas apontaram escores baixos ( 1 a 3 , ou seja, muito baixo; baixo, e médio) em quase todas as atividades listadas nas variáveis que tratam de questões atuais, e escores mais elevados ( 3 a 5, ou seja, médio, alto e muito alto) para aquelas variáveis que tratavam da percepção futura, representadas pelas cores verde e amarela, de menor pontuação no presente, e cores vermelhas, de maior pontuação para futuro.

Figura 5. Análise de correspondência entre nível de capacitação exigido no futuro e a contribuição do desenvolvimento científico e tecnológico.

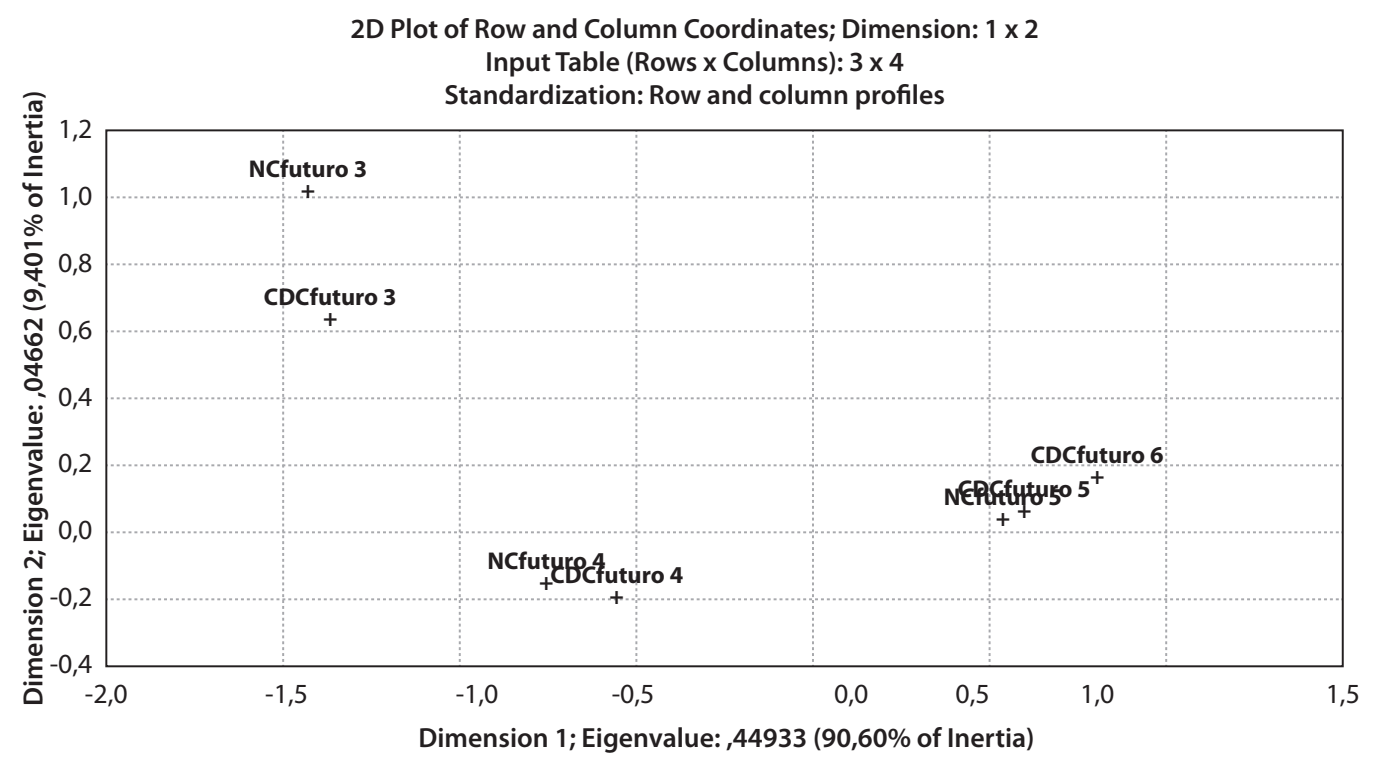

Legenda: NCfuturo - necessidade de capacitação (no futuro); CDCfuturo - contribuição do desenvolvimento científico (no futuro).

Fonte: Resultados da pesquisa. 
Figura 6. Gráfico Two-way Joinning entre atividades apontadas no painel de especialistas e variáveis analisadas.

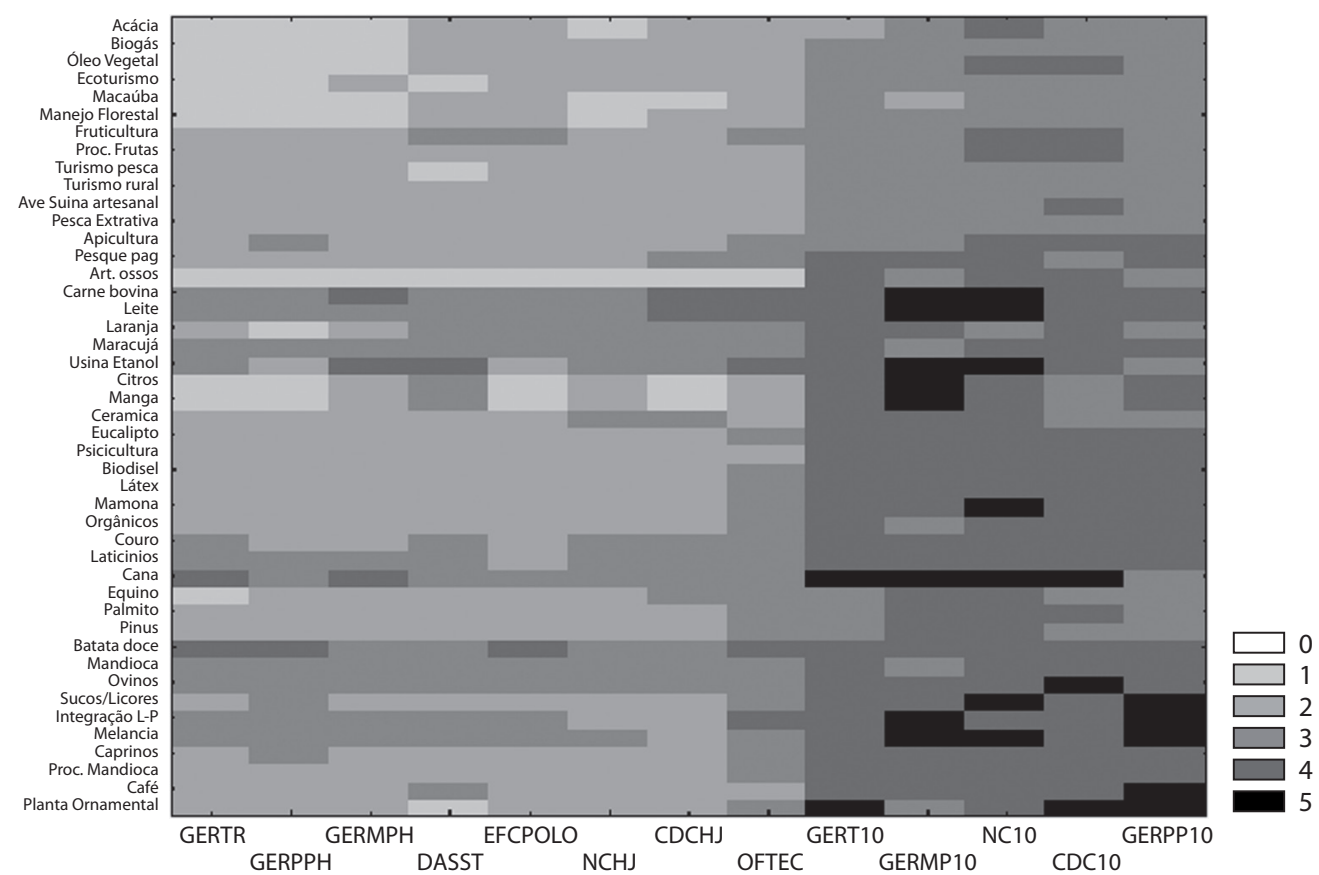

Legenda: GETR - geração de emprego ao trabalhador rural; GERT10 - geração de emprego ao trabalhador rural (no futuro); GRPPH - geração de renda ao pequeno produtor; GRPP10 - geração de renda ao pequeno produtor (no futuro); GRMPH - geração de renda ao médio e grande produtor; GRMP10 - geração de renda ao médio e grande produtor (no futuro); DASST - disponibilidade de assistência técnica; EFCPOLO - eficiência do polo em transferência de tecnologias; OFTEC - oferta de tecnologias; $\mathrm{CDCHJ}$ - contribuição do desenvolvimento científico e tecnológico; CDC10 - contribuição do desenvolvimento científico e tecnológico (no futuro); NCHJ - necessidade de capacitação; NC10 - necessidade de capacitação (no futuro).

Fonte: Resultados da pesquisa.

Isso indica um longo caminho a ser percorrido pelas diferentes instituições que compõem o ambiente de geração e transferência de tecnologias do Pontal do Paranapanema (Figura 6).

Tambémépossívelverificarqueosespecialistas da região reconhecem a eficiência do polo regional da Apta na transferência de tecnologias nas atividades agrícolas (EFCPOLO) de batata-doce (grau de importância 3,345), seguida de maracujá e laranja (grau de importância 2,927). Por outro lado, a variável "oferta atual de tecnologias" (OFTEC) tem pesos maiores que os derivados do polo em diversas atividades, indicando que tal oferta é proveniente de outras fontes e instituições. Apenas a batata-doce, maracujá e laranja receberam o mesmo peso nessas duas variáveis, indicando em quais atividades o polo regionalcontribui efetivamente para oferta de tecnologias noPontal do Paranapanema.

\subsection{Análise indicativa}

As variáveis referentes à capacidade futura para geração de emprego e renda ao trabalhador rural e pequeno produtor rural foram uilizadas para, através da análise de agrupamento (técnica k-means - Figura 7), selecionar atividades rurais mais importantes entre 45 listadas 8 . Foram obtidos dois clusters compostos, dos quais o primeiro com 18 atividades de menor capacidade de geração de empregos e renda $(2,6)$ e o segundo composto por 27 atividades com média/alta capacidade de geração de renda $(3,6)$.

8 Estas atividades foram indicadas pelos participantes do painel estruturado. 
Figura 7. Gráfico de formação de clusters pela técnica de agrupamento k-means.

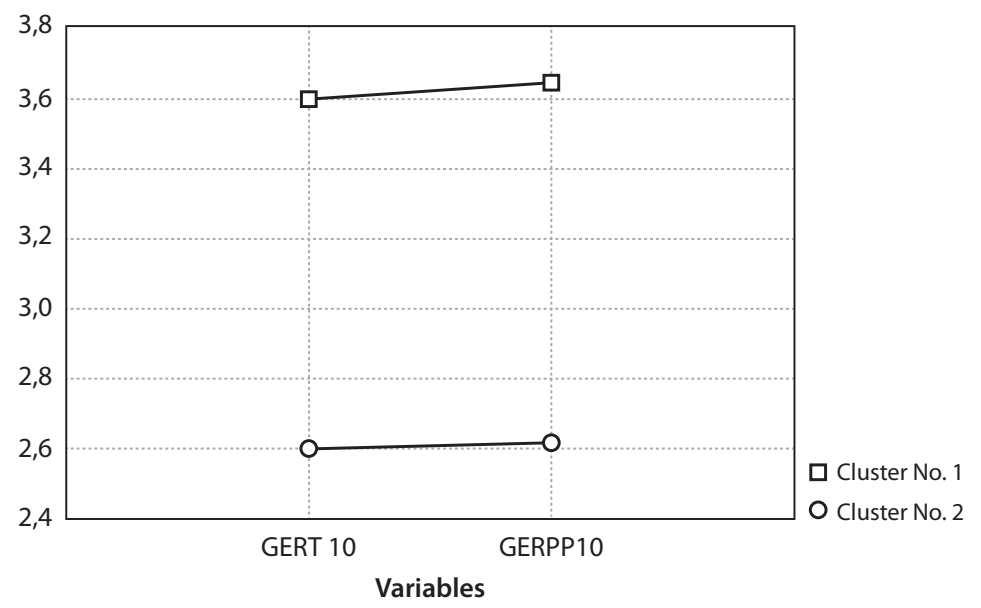

Fonte: Resultados da pesquisa.

Avaliando apenas as atividades que constituem o cluster de número 2, pode-se notar que, apesar da seleção das variáveis ponderarem a pontuação dada em cada uma das atividades, nem sempre uma mesma atividade tem o mesmo grau de capacidade para geração de empregos e renda para as duas variáveis analisadas. A atividade "usinas de cana-de-açúcar", por exemplo, recebeu um peso muito maior para a geração de emprego ao trabalhador rural do que para a geração de renda ao pequeno produtor (Tabela 4).

Nota-se a importância dada às atividades de produção relacionadas à agroindústria: a criação de bovinos de leite com a existência de laticínios na região, bovinos de corte e couro, mandioca e o seu processamento, cana-de-açúcar e as usinas de etanol e açúcar, a mamona e o biodisel, a produção de orgânicos e frutas e bebidas e alimentos diferenciados.

Essa relação tem sentido quando pensada na importância para a geração de empregos para o trabalhador rural, seja no campo, atuando diretamente na produção, ou mesmo inserido no processo de agroindustrialização. Este cenário demanda iniciativas governamentais para $\mathrm{o}$ estímulo ao crescimento do setor industrial, tão baixo na região ( $0,6 \%$ do estado), e alternativas para a pequena produção com o crédito Fundo de Expansão do Agronegócio Paulista (Feap) para agroindústria.

Outrasatividades revelamimportância quando inseridas em novos sistemas de produção, como, por exemplo, heveicultura, eucalipto, caprinos e ovinos, integração lavoura-pecuária,piscicultura e pesque-pague, direcionados para a geração de renda para o pequeno produtor rural e, principalmente, a atividade de produção de plantas ornamentais, considerada de alta capacidade para a geração de renda, tendo em vista a dependência da Região Administrativa de Presidente Prudente (53 municípios) em relação a produtos oriundos de outras localidades, tais como Holambra (I e II) e Ceagesp, na capital paulista.

Algumas das atividades mais importantes atualmente para a região e mais expressivas em relação ao estado de São Paulo permaneceram destacadas no horizonte de 10 anos: batata-doce, carne bovina, melancia, mandioca e leite bovino.

As atividades de batata-doce, maracujá e laranja, que recebem maior contribuição tecnológica do polo regional da Apta, também estão incluídas na lista das 27 atividades prioritárias (Tabela 4).

Cabe acrescentar que a indicação de grande número de atividades potenciais está de acordo 
Tabela 4. Cluster 2: atividades rurais com maior potencial para a geração de renda a pequenos produtores rurais e geração de empregos para trabalhadores rurais, no horizonte de 10 anos (2008-2018).

\begin{tabular}{|c|c|c|c|}
\hline Atividades & $\begin{array}{l}\text { Geração de renda para o pe- } \\
\text { queno produtor em } 10 \text { anos }\end{array}$ & $\begin{array}{c}\text { Geração de emprego para o } \\
\text { trabalhador em } 10 \text { anos }\end{array}$ & Classificação \\
\hline Plantas Ornamentais & 4,3 & 4,1 & 2,0 \\
\hline Café & 4,2 & 3,7 & 3,0 \\
\hline Integração Lavoura/Pecuária & 4,1 & 3,8 & 5,0 \\
\hline Melancia & 4,0 & 3,6 & 2,0 \\
\hline Fecularia de mandioca & 4,0 & 3,9 & 4,0 \\
\hline Alimentos diferenciados & 4,0 & 3,9 & 4,0 \\
\hline Bovino de Leite & 3,9 & 4,0 & 1,0 \\
\hline Batata-doce & 3,9 & 3,8 & 2,0 \\
\hline Laticínios & 3,9 & 3,5 & 4,0 \\
\hline Caprinocultura & 3,8 & 3,7 & 1,0 \\
\hline Manga & 3,8 & 3,7 & 3,0 \\
\hline Citros & 3,8 & 3,7 & 3,0 \\
\hline Ovinocultura de corte & 3,6 & 3,6 & 1,0 \\
\hline Mandioca & 3,6 & 3,5 & 2,0 \\
\hline Heveicultura & 3,6 & 3,4 & 3,0 \\
\hline Biodiesel & 3,6 & 3,3 & 4,0 \\
\hline Orgânicas & 3,6 & 3,5 & 5,0 \\
\hline Apicultura & 3,5 & 3,0 & 1,0 \\
\hline Piscicultura & 3,4 & 3,3 & 1,0 \\
\hline Maracujá & 3,4 & 3,2 & 2,0 \\
\hline Bovino de Corte & 3,3 & 3,3 & 1,0 \\
\hline Eucalipto & 3,3 & 3,2 & 3,0 \\
\hline Mamona & 3,3 & 3,2 & 3,0 \\
\hline Pesque-Pague & 3,3 & 3,0 & 6,0 \\
\hline Couro & 3,2 & 3,8 & 4,0 \\
\hline Cana-de-açúcar & 2,9 & 4,1 & 3,0 \\
\hline Usinas de etanol/açúcar & 2,8 & 3,8 & 4,0 \\
\hline
\end{tabular}

Nota: Classificação: Pecuária (1); Agrícultura temporária (2); Agricultura permanente (3); Agroindústria (4); Processos de produção (5); Serviços (6). Fonte: Resultados da pesquisa.

com o que foi apresentada por Veiga (2001), sendo adiversificação produtiva a estratégia mais interessante para o desenvolvimento econômico regional, sendo necessário dispor de condições favoráveis de comunicações, serviços e, sobretudo, para o desenvolvimento de uma cultura de empreendedorismo e inovação.

Por outro lado, a importância de tais atividades no horizonte futuro (10 anos) abre a possibilidade de realização de pesquisa aplicada e desenvolvimento de produtos e processos inovadores na região, durante esse período, em diversas áreas.Tal estratégia permitiria subsidiar, por exemplo, a adaptação de materiais genéticos à região, em função das características climáticas, e mesmo viabilizar vantagens comparativas como a disponibilidade de grandes áreas de pastagens (como no caso da produção total de ovinos em pastejo), bem como a disponibilidade de mãodeobra (heveicultura ou produção de algodão colorido para posterior tecelagem).

Entretanto, a ação isolada de geração de P\&D para as atividades listadas não garante a adoção de tais tecnologias pelo público-alvo, mesmo quando alicerçadas por processos participativos de identificação de necessidades em inovação em produtos e processos. A ação não é linear nem imediata (KLINE e ROSENBERG 1986) e exige a interação entre os diversos atores, de forma participativa em todo o processo, desde a identificação das demandas, o desenvolvimento de pesquisas, a validação dessa pesquisa até a sua adoção com o intuito de solucionar os problemas regionais. 


\section{Conclusões}

- As atividades de produção, transformação e serviços com potencial futuro para geração de empregos e renda exigirão maiores esforços de P\&D e Transferência de Tecnologias para que possam de fato gerar emprego e renda futuramente;

- Alguns problemas produtivos identificados poderiam ser solucionados através de ações de transferência de novas tecnologias e inovações adaptadas às condições regionais;

- A função de P\&D com foco na inovação tecnológica em produtos e processos poderia ser atribuída ao Polo Regional da Alta Sorocabana (APTA), principalmente em atividades agropecuárias que façam parte de sistemas produtivos menos organizados;

- Ë necessária a criação de condições internas propícias no Polo Regional da Alta Sorocabana, através de: modernização da infraestrutura, agregação de novos pesquisadores, recomposição do quadro técnico de apoio, melhorias na gestão da unidade e o desenvolvimento de cultura científica e tecnológica voltada à inovação;

- A formulação de políticas institucionais (DDD/APTA e APTA/SAA-SP) para oferecimento de capacitação técnica aos trabalhadores da região, variável relacionada com as ações de P\&D, é fundamental para transformação de potencial em realidade;

- São necessárias políticas setoriais de estímulo à agroindustrialização, que demandarão esforços conjuntos com outras instituições regionais ligadas a esse processo, avaliações de viabilidade técnica e estratégicas geográficas, além da adequação de hábitos e rotinas de trabalho buscando atender satisfatoriamente essas necessidades.

\section{Referências bibliográficas}

ALEIXO, S.S., SOUZA. J.G. e FERRAUDO, A.S. Técnicas de análise multivariada na determinação de grupos homogêneos de produtores de leite. Revista Brasileira de Zootecnia, v.36, n.6, p.2168-2175, 2007.

BENZECRI, J. P. Analyse de données: analyse de correspondances. Paris: Dunod, 1973.

BLISKA, F. M. M., GUERREIRO FILHO, O., FAZUOLI, L. C., SILVAROLLA, M. B. e GONÇALVES, W. Priorização de estratégias de pesquisa e desenvolvimento para a cadeia de produção do café no Estado de São Paulo. Agricultura em São Paulo, São Paulo, v. 52, n. 2, p. 29-45, 2005.

BLISKA, F. M. M., RAZOOK, A. G., PITUCO, E. M., ALLEONI, G. F., COUTINHO, J. L. V., GONÇALVES, J. R. e LEME, P. R. Prospecção de demandas tecnológicas na cadeia produtiva da carne bovina no Estado de São Paulo. Boletim do Instituto de Zootecnia, Nova Odessa, v. 42, p. $1-71,1998$.

BLISKA, F. M. M. (Org.) e GUERREIRO FILHO, O. (Org.). Prospecção de demandas na cadeia produtiva do café no Estado de São Paulo. 1. ed. Campinas: Instituto Agronômico, 2007. v. 1.75 p.

BOUROCHE, J.M. e SAPORTA, G. Análise de dados. Tradução de: M. Penchel. Rio de Janeiro: Zahar Editores, 116 p., 1980.

CAPANEMA L.M., COMITRE, V., ROSA, F. F,, VASCONCELOS, L. G., CARMO, M. S. e OLIVERIA, J. T. A. Prospecção de Demandas Tecnológicas para o Pólo Regional do Vale do Paraíba da Agência Paulista de Tecnologias dos Agronegócios. In: Congresso Abipti 2006, Campinas. Competitividade e riqueza nacional: o futuro das Instituições de Pesquisa Tecnológica no Sistema Nacional de Ciência, Tecnologia e Inovação. Brasília: ABIPTI, 2006.

DAVIS, J. H. e GOLDEBERG, R. A Concept of Agribusiness. Boston: Havard University, 1957.

FUENTES LLANILLO, R., GROSSI, M.E.D., SANTOS, F.O., MUNHOS, P.D. e GUIMARÃES, M.F. Regionalização da agricultura do estado do Paraná. Ciência Rural, v.36, n.1, p.120-127, 2006.

GODET, M. A. Caixa de Ferramentas da prospectiva tecnológica. Centro de Estudos de Prospectiva e Estratégia - CEPES, Lisboa, 2000.

HAIR, J.F., TATHAM, R.L., ANDERSON, R.E. e BLACK, W.C.; trad. Adonai Schlup Sant'Anna e Anselmo 
Chaves Neto. Análise multivariada de dados. Porto Alegre: Bookman, 5a ed., 2005.

HOFFMANN, R. A dinâmica da modernização da agricultura em 157 microrregiões homogêneas do Brasil. Revista de Economia e Sociologia Rural, v. 30, n. 4, p.271-290, 1992.

HORTON, A. A Simple Guide to Successful Foresight. Foresight, v. 1, n. 1, p. 5-9, 1999.

JANTSCH, E. (1967). Technological Forecasting in Perspective: a Framework for Technological Forecasting, its Techniques and Organisation. OCDE, 401p. Disponível em: <http://www.cnam.fr/lipsor/recherche/laboratoire/ memoireprospective.php $>$.

KLINE, S. e ROSENBERG, N. An Overview of Innovation. In: LANDAU, R. e ROSENBERG, N. (orgs.). The Positive Sum Strategy, Washington, DC: National Academy of Press, 1986.

LOURENÇO, A. e MATIAS, R. P. Estatística Multivariada. Instituto Superior de Engenharia do Porto, 2000.

NICHOLS, D. P. "My Coefficient - is Negative!". SPSS Keywords,n. 68, 1999. Disponível em: <http://www. ats.ucla.edu/stat/spss/library/negalpha.htm >. Acesso em: out. 2007.

NOGUEIRA E. A. e MELLO N. T. C. Pesquisa prospectiva de demanda regional no sudoeste paulista. Informações Econômicas, São Paulo, v.31, n.2, 2001.

NOGUEIRA E. A., MELLO N. T. C., CASTRO J. L., SANNAZZARO A. M. e LEINZ F. F. Pesquisa e Desenvolvimento: prospectando demandas para a região sudoeste do Estado de São Paulo. Agricultura em São Paulo, São Paulo, v. 52, n. 1, p. 63-75, 2005.
ROCHA M.B., VICENTE, M.C.M., MARTIN, N.B., OLIVEIRA, M.D.M., SILVA, V. e OTANI, M.N. Plano de desenvolvimento regional sustentável do vale do Médio Paranapanema: Estratégia de Desenvolvimento. São Paulo: 2005. Disponível em: <http://www.rlc.fao.org/es/ desarrollo/fao-bid/des/pdf/saopaulo.pdf $>$. Acesso em: 03 fev. 2010.

ROCHA, A. D. et al. Qualidade de vida, ponto de partida ou resultado final?. Ciênc. saúde coletiva, Rio de Janeiro, v. 5, n. 1, 2000.

SÃO PAUlO (ESTADO). Projeto LUPA 2007/2008: Levantamento censitário de unidades de produção agrícola do Estado de São Paulo. São Paulo: CATI/IEA/SAA, 2009. Disponível em: <http://www.cati.sp.gov.br/ projetolupa $>$. Acesso em: 2009.

- Secretaria de Estado de Economia e Planejamento - Perfil Regional da Região Administrativa de Presidente Prudente. São Paulo: SEADE, 2008.

SILVA, E. e VERDINELLI, M. A. Avaliação em massa de terrenos em Blumenau Santa Catarina - Brasil usando análises fatorial de correspondência e regressão múltipla. Textos para Discussão, UFSC CSE / Departamento de Ciências Econômicas, n. 9, 1997.

STATSOFT, INC. (1997). STATISTICA for Windows [Computer program manual]. Tulsa, OK: StatSoft, Inc., 2300 East 14th Street, Tulsa, OK 74104, phone: (918) 749-1119, fax: (918) 749-2217, email: info@statsoft.com, WEB: http://www.statsoft.com

VEIGA, J.E. O Brasil rural não encontrou seu eixo de desenvolvimento. In: EstudosAvançados. São Paulo: EDUSP, v. 15, n .43, p. 101-119, 2001. 